Supporting Information for

\title{
Probing the synergistic ratio of P450/CPR to improve (+)- nootkatone production in Saccharomyces cerevisiae
}

Yaping Cha ${ }^{\mathrm{a}}$, Wen $\mathrm{Li}^{\mathrm{a}}$, Tao $\mathrm{Wu}^{\mathrm{a}}$, Xia You ${ }^{\mathrm{a}}$, Hefeng Chen ${ }^{\mathrm{a}}$, Chaoyi Zhu ${ }^{\mathrm{a}}$, Min Zhuo ${ }^{\mathrm{a}}$, Bo Chen ${ }^{\mathrm{b}}$, and Shuang $\mathrm{Li}^{\mathrm{a} *}$

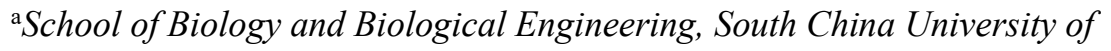

Technology, Guangzhou 510006, China

${ }^{\mathrm{b}}$ Heilongjiang Feihe Dairy Co., Ltd., Beijing 100015, China

\section{*Corresponding authors:}

Shuang Li, School of Biology and Biological Engineering, South China University of Technology, Higher Education Mega Center, Guangzhou 510006, China, E-mail: shuangli@scut.edu.cn, Tel/Fax: +862039380629

Bo Chen, Heilongjiang Feihe Dairy Co., Ltd., Star City, 10 Jiuxianqiao Rd., Beijing 100015, China, E-mail: chenbo@feihe.com, Tel: +861064317668 


\section{Contents}

Figure S1. Multiple-sequence alignments of the native $H X T 7$ promoter $\left(\mathrm{P}_{H X T 7}\right)$ and the 8 promoter

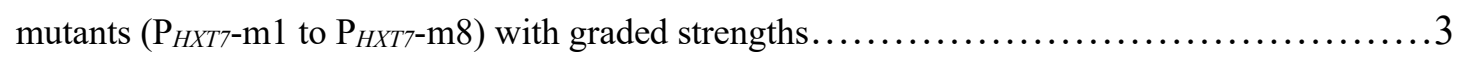

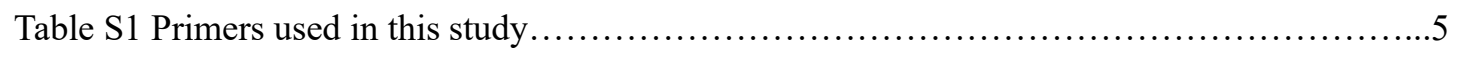

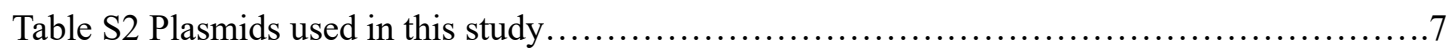

Table S3 Strains used in this study...................................................... 
folds $\quad-389$

$\mathbf{P}_{\text {HXT7 }}$

$\mathrm{P}_{\text {HXT7 }}-\mathrm{m} 1$

$\mathrm{P}_{\text {HXT7 }}-\mathrm{m} 2$

$\mathrm{P}_{\mathrm{HXT} 7}-\mathrm{m} 3$

$\mathrm{P}_{\mathrm{HXT7}}-\mathrm{m} 4$

$\mathrm{P}_{\mathrm{HXT7}}-\mathrm{m} 5$

$\mathrm{P}_{\text {HXT7 }}-\mathrm{m} 6$

$\mathrm{P}_{\text {HXT7 }}-\mathrm{m} 7$

$\mathrm{P}_{\text {HXT7 }}-\mathrm{m} 8$

$\mathbf{P}_{\text {HXT7 }}$

$\mathrm{P}_{\text {HXT7 }}-\mathrm{m} 1$

$\mathrm{P}_{\mathrm{HXT} 7} \mathrm{-m} 2$

$\mathrm{P}_{\mathrm{HXT} 7}-\mathrm{m} 3$

$\mathrm{P}_{\mathrm{HXT} 7}-\mathrm{m} 4$

$\mathrm{P}_{\text {HXT7 }}-\mathrm{m} 5$

$\mathrm{P}_{\text {HXT7 }}-\mathrm{m} 6$

$\mathrm{P}_{\mathrm{HXT} 7}-\mathrm{m} 7$

$\mathrm{P}_{\text {HXT7 }}-\mathrm{m} 8$

$\mathrm{P}_{\text {HXT7 }}$

$\mathrm{P}_{\text {HXT7 }}-\mathrm{m} 1$

$\mathrm{P}_{\mathrm{HXT} 7} \mathrm{-m} 2$

CTCGTAGGAAATTTCGGGCCCCTGCGTGTTCTTCTGAGGTTCATCTTTTACATTTGCTTCTGCTGGATAATTTTCAGAGGCAACAAGGAAAAATTAGATGGCAAAAAGTCGTCTTTCAAG

CTCGTAGGAAATTTCGGGCCCCTGCGTGTTCTTCTGAGGTTCATCTTTTACATTTGCTTCTGCTGGATAATTTTCAGAGGCAACAAGGAAAAATTAGATGGCAAAAAGTCGTCTTTCAAG CTCGTAGGAAATTTCGGGCCCCTGCGTGTTCTTCTGAGGTTCATCTTTTACATTTGCTTCTGCTGGATAATTTTCAGAGGCAACAAGGAAAAATTAGATGGCAAAAAGTCGTCTTTCAAG CTCGTAGGAAATTTCGGGCCCCTGCGTGTTCTTCTGAGGTTCATCTTTTACATTTGCTTCTGCTGGATAATTTTCAGAGGCAACTAGGAAAAATTAGATGGCAAAAAGTCGTCCTTCAAG CTCGTAGGAAATTTAGGGCCCCTGCGTGTTCTTCTGAGGTTCATCTTTTACATTTGCTTCTGCTGGATAATTTTCAGAGGCAACAAGGAAAAATTAGATGGCAAAAAGTCGTCTTTCAAG CTCGTAGGAAATTTCGGGCCCCTGCGTGTTCTTCTGAGGTTCGTCTTTTACATTTGCTTCTGCTGGATAATTTTCAGAGGCAACAAGGAAAAATTAGATGGCAAAAAGTCGTCTTTCAAG CTCATAGGAAATTTCGGGCCCCTGCGTGTTCTTCTGAGGTTCATCTTTTACATTTGCTTCTGCTGGATAATTTTCAGAGGCAACAAGGAAAAATTAGATGGCAAAAAGTCGTCTTTCAAG CTCGTAGGAAATTTCGGGCCCCTGCGTGTTCTTCTGAGGTTCATCTTTTACATTTGCTTCTGCTGGATAATTTTCAGAGGCAACAAGGAAAAATTAGATGGCAAAAAGTCGTCTTTCAAG CTCGTAGGAATTTCGGGCCCCTGCGTGTTCTTCTGAGGTTCATCTTTTTCATTTGCTCCTGCTGGATAATTTTCAGAGGCAACAAGGAAAAATTAGATGGCAAAAATCGTCTTTCAAG

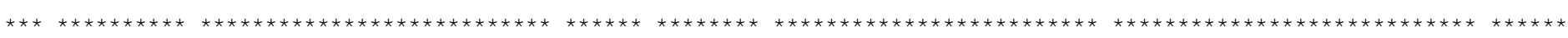

Stb5

Cup2

GAAAAATCCCCACCATCTTTCGAGATCCCCTGTAACTTATTGGCAACTGAAAGAATGAAAAGGAGGAAAATACAAAATATACTAGAACTGAAAAAAAAAAA--GTATAAATAGAGACGAT GAAAAATCCCCACCATCTTTCGAGATCCCCTGTAACTTATTGGCAACTGAAAGAATGAAAAGGAGGAAAATACAAAATATACTAGAACTGAAAAAAAAAAA--GTATAAATAGAGACGAT GAAAATCCCCACCATCTTTĀGAGATCCCCTGTAACTTATTGGCAACTGAAAGAATGAAAAGGAGGAAAATACAAAATATACTAGAACTGAAAAAAAAAAA--GTATAAATAGAGACGAT GAAAATCCCCACCATCTTTCGAGATCCCCTGTAACTTATTGGCAACTGAAAGAATGAAAAGGAGAAAAATACAAAATAAACTAGAACTGAAAAAAAAAAAAAGTATAAATAGAGACGAT GAAAAATCCCCACCATCTTTCGAGATCCCCTGTAACTTATTGGCAACTGAACGAATGAAAAGGAGGAAAATACAAAATATACTAGAACTGAAAAAAAAAAA--GTATAAATAGAGACGAT GAAAAATCCCCACCATCTTTCGAGATCCCCTGTAACTTATTGGCAACTGAAAGAATGAAAAGGAGGAAAATACAAAATATACTAGAACTGAAAAAAAAAAAA-GTATAAATAGAGACGAT GAAAAATCCCCACCATCTTTCGAGATCCCCTGTTACTTATTGGCAACTGAAAGAATGAAAAGGAGGAAAATACAAAATATACTAGAACTGAAAAAAAAAAA--GTATAAATAGAGACTAT GAAAAATCCCCACCATCTTTCGAGATCCCCTGTAACTTATTGGCAACTGAAAGAATGAAAAGGAGGAAAATACAAATTATACTAGAACTGAAAAAAAAAAA--GTATAAATAGAGACGAT GAAAAATCCCCACCATCTTTCGAGATCCCCTGTAACTTATTGGCAACTGAAAGAATGAAAAGGAGGAAATACAAAATATACTAGAACTGAAAAAAAAAA---GTATAAATAGAGACGAT

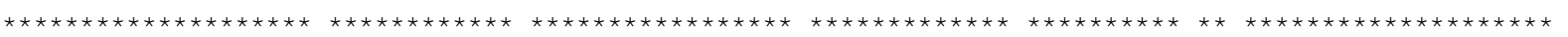

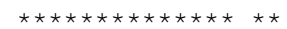

CCAAT bOX

Tec1

Mot2

TATA bOX Arg81 ATATGCCAATACTTCACAATGTTCGAATCTATTCTTCATTTGCAGCTATTGTAAAATAATAAAACATCAAGAACAAACAAGCTCAACTTGTCTTTTCTAAGAACAAAGAATAAACACAAA ATATGCCAATACTTCACAATGTTCGAATCTATTCTTCATTTGCAGCTATTGTAAA GTAATAAAACATCAAGAACAAACAAGCTCAACTTGTCTTTTCTAAGAACAAAGAATAAACACAAA ATATGCCAATACTTCACAATGTTCGAATCTATTCTTCATTTGCAGCTATTGTAAAATAATAAAACATCAAGAACAAACAAGCTCAACTTGTCTTTTCTAAGAACAAAGAATAAACACAAA 
$\mathrm{P}_{\mathrm{HXT7}}-\mathrm{m} 3$

$\mathrm{P}_{\mathrm{HXT7}}-\mathrm{m} 4$

$\mathbf{P}_{\text {HXT7 }}-\mathrm{m} 5$

$\mathrm{P}_{\text {HXT7 }}-\mathrm{m} 6$

$\mathrm{P}_{\mathrm{HXT} 7}-\mathrm{m} 7$

$\mathrm{P}_{\text {HXT7 }}-\mathrm{m} 8$

$\mathbf{P}_{\text {HXT7 }}$

$\mathrm{P}_{\mathrm{HXT7}}-\mathrm{m} 1$

$\mathrm{P}_{\mathrm{HXT} 7}-\mathrm{m} 2$

$\mathrm{P}_{\text {HXT7 }}-\mathrm{m} 3$

$\mathrm{P}_{\mathrm{HXT7}}-\mathrm{m} 4$

$\mathrm{P}_{\text {HXT7 }}-\mathrm{m} 5$

$\mathrm{P}_{\text {HXT7 }}-\mathrm{m} 6$

$\mathrm{P}_{\text {HXT7 }}-\mathrm{m} 7$

$\mathrm{P}_{\mathrm{HXT7}}-\mathrm{m} 8$
ATATGCCAATACTTCACAATGTTCGTATCTATTCTTCATTTGCAGCTATTGTAAAATAATAAAACATCAGGAACAAACAAGCTCAACTTGTCTTTTCTAAGAACAAAGAATAAACACAAA ATATGCCAATACTTCACAATGTTCGAATCTATTCTTCATTTGCAGCTATTGTAAAATAATAAAACATCAAGAACAAACAAGCTCAACTTGTCTTTTCTAAGAACAAAGAATAAACACAAA ATATGCCAATACTTCACAATGTTCGAATCTATTCTTCATTTGCAGCTATTGTAAAATAATAAAACATCAAGAACAAACAAGCTCAACTTGTCTTTTCTAAGAACAAAGAATAAACACAAA ATATGCCAATACTTCACAATGTTCGAATCTATTCTTCATTTGCAGCTATTGTAAAATAATAATACATCAAGAACAAACAAGCTCAACTTGTCTTTTCTAAGAACAAAGAATAAACACAAA ATATGCCAATACTTCACAATGTTCAAATCTATTCTTCATTTGCAGCTATTGTAAAATAATAAAACATCAAGAACAAACAAGCTCAACTTGTCTTTCCTAAGAACAAAGAATAAACACAAA ATATGCCAATACTTCACAATGTTCGAATCTATTCTTCATTTGCAGCTATTGTAAAATAATAAAACATCAAGAACAAACAAGCTCAACTTGTCTTTTCTAAGAACAAAGAATAAACACAAA

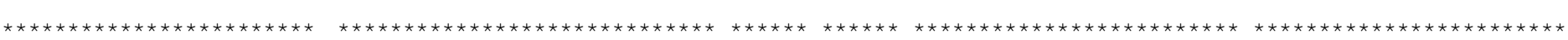

Pho2 Ste12

\section{AACAAAAAGTTTTTTTTAATTTTAATCAAAAA}

AACAAAAAGTÄTTTTTAATTTTAATCAAAAA

ACCAAAA-GTTTTTTTAATTTTAATCAAAAA

AACAAAAAGTTTTTTTAATTTTAATCAAAAA

AACAAAAAGTTTTTTTAATTTTAATCAAAAA

AACAAAAAgTTTTTTTAATTTTAATCAAAAA

AACAAAAAGTTTTTTTIATTTAAATCAAAAA

AACAAAAAGTTTTTTTAATTTTAATCAAAAA

AACAAAAAGTTTTTTTAATTTTAATCAAAAA

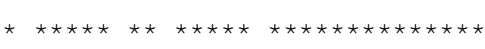

Fkh1/2 Pho2 -1

Figure S1. Multiple-sequence alignments of the native $H X T 7$ promoter $\left(\mathrm{P}_{H X T 7}\right)$ and the 8 promoter mutants $\left(\mathrm{P}_{H X T 7-\mathrm{m} 1 \text { to }} \mathrm{P}_{H X T 7}-\mathrm{m} 8\right)$ with graded strengths. The normalized promoter strength for different promoters were presented at the beginning as folds. The underlined sequences indicate the predicted transcription factor binding sites. 
Table S1 Primers used in this study ${ }^{a}$

\begin{tabular}{|c|c|}
\hline Primers & Sequence $\left(5^{\prime}-3^{\prime}\right)$ \\
\hline HP-F & AAGCTTGGCACTGGCCGTCGTTTTACAACGTC \\
\hline AT-R & CGACGGCCAGTGCCAAGCTTCTATATAACAGTTGAAATT \\
\hline $\mathrm{L}_{\mathrm{GGGS}}-\mathrm{F}$ & $\begin{array}{l}\text { CTTATCAACCCTCCCGTGAAGGTGGCGGTTCTACTTCTGCCTTGT } \\
\text { ATGCCT }\end{array}$ \\
\hline $\mathrm{L}_{\mathrm{GGGS}}-\mathrm{R}$ & $\underline{\text { AGAACCGCCACCTTCACGGG }}$ \\
\hline $\mathrm{Lt}_{\mathrm{GGGS}}-\mathrm{F}$ & CCCGTGAAGGTGGCGGTTCTTGGAAAAAGACCACCGCC \\
\hline $\mathrm{Lt}_{\mathrm{GGGS}}-\mathrm{R}$ & $\underline{\text { AGAACCGCCACCTTCACGGG }}$ \\
\hline $\mathrm{Lt}_{\text {EAAAK }}-\mathrm{F}$ & GTGAAGAAGCAGCTGCAAAATGGAAAAAGACCACCGCC \\
\hline $\mathrm{Lt}_{\text {EAAAK }}-\mathrm{R}$ & TTTTGCAGCTGCTTCTTCACGGG \\
\hline HPO-F & CTGTCCTTTCTGTTCCTGCTGAGG \\
\hline HPO-R & ACATGATGAGGCAATCCACCAACC \\
\hline AtCPR-F & CCACCACCATTTCCAGGTCCATG \\
\hline AtCPR-R & GCAGCCAAAGCAACTAAAGCAGAC \\
\hline gSEDl-F & AGAGGAGGAAGTGACATCGGGTTTTAGAGCTAGAAATA \\
\hline gSED1-R & CCGATGTCACTTCCTCCTCTGATCATTTATCTTTCACTGC \\
\hline Tong-F & TAATAATGGTTTCTTAGTATGA \\
\hline Tong-R & ACTAAGAAACCATTATTATCAT \\
\hline donor-F & $\begin{array}{l}\text { TACTTTGGCCCAATTTTCCAACAGTACATCTGCTTCTTAAACCGA } \\
\underline{\text { TGTCACTTCCTCCT }}\end{array}$ \\
\hline donor-R & $\begin{array}{l}\text { TGTGATAGTTACTGAGCCAGAGGAAGTGGAGATGGAAGAGGAG } \\
\text { GAAGTGACATCGGTTT }\end{array}$ \\
\hline Ysed1-F & СССТCTTTTGAACTGTCATA \\
\hline Ysedl-R & GTAGTTGGTGGGAAAGCTGA \\
\hline GUS-F & ATAATTAACT AAACTCTAGAATGTTACGTCCTGTAGAAACCC \\
\hline GUS-R & ACATGATGCGGCCCGTCGACTCATTGTTTGCCTCCCTGCT \\
\hline$H X T 7-F$ & ACAAAAGCTGGTCGACCT \\
\hline$H X T 7-R$ & ACGTAACATGAGCTCCTG \\
\hline$H X T 7 \mathrm{~s}-\mathrm{F}$ & GCAACGCAATTAAGTG \\
\hline HXT7-F1 & TTTACAGGATCCCTCGTAGGAAAAATTTCGGG \\
\hline$H X T 7-\mathrm{F} 2$ & TTTACAGGATCCCTCGTAGGAAAAATTTAGGG \\
\hline$H X T 7-\mathrm{F} 3$ & TTTACAGGATCCCTCATAGGAAAAATTTCGGG \\
\hline$H X T 7-\mathrm{F} 4$ & GAGCGAGAATTCCTCGTAGGAAAAATTTCGGG \\
\hline$H X T 7-\mathrm{F} 5$ & GAGCGAGAATTCCTCGTAGGAAAAATTTAGGG \\
\hline HXT7-F6 & GAGCGAGAATTCCTCATAGGAAAAATTTCGGG \\
\hline$H X T 7-\mathrm{R} 1$ & GAGCGATCTAGATTTTTGATTAAAATTAAAAAAAC \\
\hline$H X T 7-\mathrm{R} 2$ & GAGCGATCTAGATTTTTGATTAAAATTAAAAATAC \\
\hline$H X T 7-\mathrm{R} 3$ & GAGCGATCTAGATTTTTGATTAAAATAAAAAAAAC \\
\hline$H X T 7-\mathrm{R} 4$ & TTTACAGAGCTCTTTTTGATTAAAATTAAAAATAC \\
\hline$H X T 7-\mathrm{R} 5$ & TTTACAGAGCTCTTTTTGATTAAAATTAAAAAAAC \\
\hline$H X T 7-\mathrm{R} 6$ & TTTACAGAGCTCTTTTTGATTAAAATAAAAAAAAC \\
\hline $\mathrm{tC}-\mathrm{F}$ & GTACCCGGGGATCCTCTAGACTTCAAAATGTTTCTACTCC \\
\hline
\end{tabular}




\begin{tabular}{|c|c|}
\hline tC-R & CCAAGCTTGCATGCCTGCAGTTTGATTATGTTCTTTCTATTTG \\
\hline HA-F & $\underline{\text { ACCCTATGGGATCCTCTAGAATGACCATGATTACGAATTC }}$ \\
\hline HA-R & AGCTTGCATGCCTGCAGCGGCCAGTGCCAAGCTT \\
\hline tC-F1 & ACCCTATGGGATCCTCTAGACTTCAAAATGTTTCTACTCC \\
\hline tC-R1 & TTTACAGTCGACTTAGGGAATAATCGGTTCGA \\
\hline \multirow{2}{*}{$E R G 20-\mathrm{F}$} & CCGTTGAACATTTCAGCCATACCAGAACCTTTGCTTCTCTTGTAA \\
\hline & $\mathrm{AC}$ \\
\hline$E R G 20-\mathrm{R}$ & CAGTCAAATCAATCAAATCTAGAATGGCTTCAGAAAAAGAA \\
\hline \multirow{2}{*}{ AtC-F } & CCGTTGAACATTTCAGCCATACCAGAACCTTTGATTGATTTGACT \\
\hline & GTGTTATTTTGC \\
\hline \multirow{2}{*}{ AtC-R } & GTTTACAAGAGAAGCAAAGGTTCTGGTATGGCTGAAATGTTCAA \\
\hline & CGG \\
\hline 414HA-F & GGGAACAAAAGCTGGAGCTCATGACCATGATTACGAATTC \\
\hline 414HA-R & TATAGGGCGAATTGGGTACCCGGCCAGTGCCAAGCTT \\
\hline$A D H 3-\mathrm{F}$ & GGGAACAAAAGCTGGAGCTCACAGTTTATTCCTGGCATCCACT \\
\hline$A D H 3-\mathrm{R}$ & CATGGCCCATAGGGTAGGGGAATTTC \\
\hline 414HA-F1 & ATTCCCCTACCCTATGGGCCATGACCATGATTACGAATTC \\
\hline InHis3-F & GAATATGTTCATAGGGTAGACGTTTTAAGAGCTTGGTGAGC \\
\hline InLeu2-R & GCTCACCAAGCTCTTAAAACGTCTACCCTATGAACATATTC \\
\hline \multirow{2}{*}{ donor062-F } & AGGAACTGCCGTCACATACGACACTGCCCCTCACGTAAGGGCAA \\
\hline & CTGTGGGAATACTCA \\
\hline \multirow{2}{*}{ donor062-R } & CCCCGAATTTATTACGAATTTGCCCACATGGTCGGTGTCGAGTTC \\
\hline & AAGAGAAAAAAAA \\
\hline Ypl062w-F & CTAAAAACCGTACTCACAAC \\
\hline Ypl062w-R & TTGTCTTGAATCCCCCTCAC \\
\hline ICE2-F & TTTACAGTCGACTCAACTACCAGAACCTATTA \\
\hline ICE2-R & GAGCGATCTAGAATGACCAGTTTGTCCAAAAGC \\
\hline \multirow{2}{*}{ donorleu2-F } & TCATCTCCGATGAAGCCTCCGTTATCCCAGGTTCCTTGGGTGAAT \\
\hline & TCGAGCTCGGTAC \\
\hline \multirow{2}{*}{ donorleu2-R } & TACAAACCAAATGCGGTGTTCTTGTCTGGCAAAGAGGCCAAGCT \\
\hline & ATGACCATGATTAC \\
\hline Leu2-F & TGAAGCCTCCGTTATCCCAG \\
\hline Leu2-R & ACCGTGGCATGGTTCGTACA \\
\hline
\end{tabular}

${ }^{\text {a }}$ The complementary sequences are underlined. 
Table S2 Plasmids used in this study

\begin{tabular}{|c|c|c|}
\hline Plasmid & Description & Source \\
\hline YEp352 & S. cerevisiae-E. coli shuttle vector, high-copy $(2 \mu), U R A 3, A m p^{\mathrm{R}}$ & Addgene \\
\hline p352-H ${ }_{\mathrm{GGGS}} \mathrm{A}$ & YEp352 derived, containing $\mathrm{P}_{H X T 7}-H P O-G G G S-A t C P R-\mathrm{T}_{T P I I}$ & This study \\
\hline p352-H GGGStA & 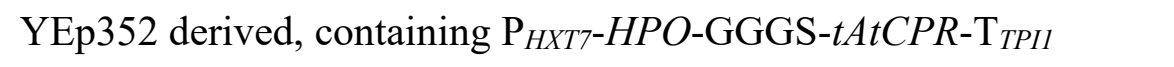 & This study \\
\hline $\mathrm{p} 352-\mathrm{H}_{\text {EAAAK }} \mathrm{tA}$ & YEp352 derived, containing $\mathrm{P}_{H X T 7}-H P O$-EAAAK-tAtCPR-T $\mathrm{T}_{T P I I}$ & This study \\
\hline p352-HA & YEp352 derived, containing $\mathrm{P}_{H X T 7}-H P O-\mathrm{T}_{T P I 1}-\mathrm{P}_{H X T 7}-A t C P R-\mathrm{T}_{T P I 1}$ & This study \\
\hline P352-GUS & YEp352 derived, containing $\mathrm{P}_{T E F 2}-G U S-\mathrm{T}_{C Y C 1}$ & This study \\
\hline $\mathrm{p} 352-\mathrm{HA} \mathrm{m} 1$ & YEp352 derived, containing $\mathrm{P}_{H X T 7}-H P O-\mathrm{T}_{T P I I}-\mathrm{P}_{H X T 7 m 1}-A t C P R-\mathrm{T}_{T P I I}$ & This study \\
\hline $\mathrm{p} 352-\mathrm{HA}_{\mathrm{m} 2}$ & YEp352 derived, containing $\mathrm{P}_{H X T 7}-H P O-\mathrm{T}_{T P I 1}-\mathrm{P}_{H X T 7 m 2}-A t C P R-\mathrm{T}_{T P I I}$ & This study \\
\hline $\mathrm{p} 352-\mathrm{HA}_{\mathrm{m} 3}$ & YEp352 derived, containing $\mathrm{P}_{H X T 7}-H P O-\mathrm{T}_{T P I 1}-\mathrm{P}_{H X T 7 m 3}-A t C P R-\mathrm{T}_{T P I I}$ & This study \\
\hline $\mathrm{p} 352-\mathrm{HA}_{\mathrm{m}}$ & YEp352 derived, containing $\mathrm{P}_{H X T 7}-H P O-\mathrm{T}_{T P I I}-\mathrm{P}_{H X T 7 m 4}-A t C P R-\mathrm{T}_{T P I I}$ & This study \\
\hline $\mathrm{p} 352-\mathrm{HA} \mathrm{m} 5$ & YEp352 derived, containing $\mathrm{P}_{H X T 7}-H P O-\mathrm{T}_{T P I 1}-\mathrm{P}_{H X T 7 m 5}-A t C P R-\mathrm{T}_{T P I I}$ & This study \\
\hline $\mathrm{p} 352-\mathrm{HA}_{\mathrm{m} 6}$ & YEp352 derived, containing $\mathrm{P}_{H X T 7}-H P O-\mathrm{T}_{T P I I}-\mathrm{P}_{H X T 7 m 6}-A t C P R-\mathrm{T}_{T P I I}$ & This study \\
\hline $\mathrm{p} 352-\mathrm{HA}_{\mathrm{m} 7}$ & YEp352 derived, containing $\mathrm{P}_{H X T 7}-H P O-\mathrm{T}_{T P I I}-\mathrm{P}_{H X T 7 m}-A t C P R-\mathrm{T}_{T P I I}$ & This study \\
\hline $\mathrm{p} 352-\mathrm{HA}_{\mathrm{m} 8}$ & YEp352 derived, containing $\mathrm{P}_{H X T 7}-H P O-\mathrm{T}_{T P I 1}-\mathrm{P}_{H X T 7 m 8}-A t C P R-\mathrm{T}_{T P I I}$ & This study \\
\hline $\mathrm{p} 352-\mathrm{H}_{\mathrm{m} 1} \mathrm{~A}$ & YEp352 derived, containing $\mathrm{P}_{H X T 7 m 1}-H P O-\mathrm{T}_{T P I 1}-\mathrm{P}_{H X T 7}-A t C P R-\mathrm{T}_{T P I I}$ & This study \\
\hline $\mathrm{p} 352-\mathrm{H}_{\mathrm{m} 2} \mathrm{~A}$ & YEp352 derived, containing $\mathrm{P}_{H X T 7 m 2}-H P O-\mathrm{T}_{T P I 1}-\mathrm{P}_{H X T 7}-A t C P R-\mathrm{T}_{T P I I}$ & This study \\
\hline $\mathrm{p} 352-\mathrm{H}_{\mathrm{m} 3} \mathrm{~A}$ & YEp352 derived, containing $\mathrm{P}_{H X T 7 m 3}-H P O-\mathrm{T}_{T P I I}-\mathrm{P}_{H X T 7}-A t C P R-\mathrm{T}_{T P I I}$ & This study \\
\hline $\mathrm{p} 352-\mathrm{H}_{\mathrm{m}} \mathrm{A}$ & YEp352 derived, containing $\mathrm{P}_{H X T 7 m 4}-H P O-\mathrm{T}_{T P I l}-\mathrm{P}_{H X T 7}-A t C P R-\mathrm{T}_{T P I I}$ & This study \\
\hline $\mathrm{p} 352-\mathrm{H}_{\mathrm{m}} \mathrm{A}$ & YEp352 derived, containing $\mathrm{P}_{H X T 7 m 5}-H P O-\mathrm{T}_{T P I 1}-\mathrm{P}_{H X T 7}-A t C P R-\mathrm{T}_{T P I I}$ & This study \\
\hline $\mathrm{p} 352-\mathrm{H}_{\mathrm{m}} \mathrm{A}$ & YEp352 derived, containing $\mathrm{P}_{H X T 7 m 6}-H P O-\mathrm{T}_{T P I I}-\mathrm{P}_{H X T 7}-A t C P R-\mathrm{T}_{T P I I}$ & This study \\
\hline $\mathrm{p} 352-\mathrm{H}_{\mathrm{m} 7 \mathrm{~A}}$ & YEp352 derived, containing $\mathrm{P}_{H X T 7 m 7}-H P O-\mathrm{T}_{T P I I}-\mathrm{P}_{H X T 7}-A t C P R-\mathrm{T}_{T P I I}$ & This study \\
\hline $\mathrm{p} 352-\mathrm{H}_{\mathrm{m} 8} \mathrm{~A}$ & YEp352 derived, containing $\mathrm{P}_{H X T 7 m 8}-H P O-\mathrm{T}_{T P I 1}-\mathrm{P}_{H X T 7}-A t C P R-\mathrm{T}_{T P I 1}$ & This study \\
\hline p352-tC & YEp352 derived, containing $\mathrm{P}_{T E F 1}-t H M G 1-\mathrm{T}_{A D H 1}-\mathrm{P}_{P D C 1}-C n V S-\mathrm{T}_{S A G 1}$ & This study \\
\hline
\end{tabular}




\begin{tabular}{|c|c|c|}
\hline p352-ADH3 & YEp352 derived, containing $\mathrm{P}_{T D H 3}-A D H 3-\mathrm{T}_{A D H 1}$ & This study \\
\hline $\mathrm{p} 352-\mathrm{AH}_{\mathrm{m}} \mathrm{A}$ & $\begin{array}{l}\text { YEp352 derived, containing } \mathrm{P}_{T D H 3}-A D H 3-\mathrm{T}_{A D H 1}-\mathrm{P}_{H X T 7 m 6}-H P O-\mathrm{T}_{T P I 1}-\mathrm{P}_{H X T 7}-A t C P R- \\
\mathrm{T}_{T P I 1}\end{array}$ & This study \\
\hline p352-AtC & $\begin{array}{l}\text { YEp352 derived, containing } \mathrm{P}_{T D H 3}-A D H 3-\mathrm{T}_{A D H 1}-\mathrm{P}_{T E F 1}-t H M G 1-\mathrm{T}_{A D H 1}-\mathrm{P}_{P D C l}-C n V S- \\
\mathrm{T}_{S A G 1}\end{array}$ & This study \\
\hline p352-AtEC & $\begin{array}{l}\text { YEp352 derived, containing } \mathrm{P}_{T D H 3}-A D H 3-\mathrm{T}_{A D H 1}-\mathrm{P}_{T E F 1}-t H M G 1-\mathrm{T}_{A D H 1}-\mathrm{P}_{P D C l}-E R G 20- \\
\text { GSG-CnVS-T } \mathrm{T}_{S A G 1}\end{array}$ & This study \\
\hline p352-ICE2 & YEp352 derived, containing $\mathrm{P}_{C Y C l}-I C E 2-\mathrm{T}_{C Y C l}$ & This study \\
\hline p414 & $\mathrm{p} 414-\mathrm{CEN} / \mathrm{ARS}-\mathrm{P}_{T E F 1}-\mathrm{Cas} 9-\mathrm{T}_{C Y C l}, T R P 1, A m p^{\mathrm{R}}$ & Addgene \\
\hline p414-GUS & $\mathrm{p} 414$ derived, $\mathrm{P}_{H X T 7-G U S-\mathrm{T}_{T P I 1}}$ & This study \\
\hline p414-GUS1 & $\mathrm{p} 414$ derived, $\mathrm{P}_{H X T 7 m I}-G U S-\mathrm{T}_{T P I I}$ & This study \\
\hline p414-GUS2 & p414 derived, $\mathrm{P}_{H X T 7 m 2-G U S-\mathrm{T}_{T P I I}}$ & This study \\
\hline p414-GUS3 & $\mathrm{p} 414$ derived, $\mathrm{P}_{H X T 7 m 3}-G U S-\mathrm{T}_{T P I I}$ & This study \\
\hline p414-GUS4 & $\mathrm{p} 414$ derived, $\mathrm{P}_{H X T 7 m 4-G U S-\mathrm{T}_{T P I I}}$ & This study \\
\hline p414-GUS5 & p414 derived, $\mathrm{P}_{H X T 7 m 5-G U S-\mathrm{T}_{T P I I}}$ & This study \\
\hline p414-GUS6 & $\mathrm{p} 414$ derived, $\mathrm{P}_{H X T 7 m \sigma^{-}} G U S-\mathrm{T}_{T P I I}$ & This study \\
\hline p414-GUS7 & $\mathrm{p} 414$ derived, $\mathrm{P}_{H X T 7 m 7-G U S-\mathrm{T}_{T P I I}}$ & This study \\
\hline p414-GUS8 & p414 derived, $\mathrm{P}_{H X T 7 m 8}-G U S-\mathrm{T}_{T P I I}$ & This study \\
\hline $\mathrm{p} 414-\mathrm{AH}_{\mathrm{m}} \mathrm{A}$ & $\mathrm{p} 414$ derived, containing $\mathrm{P}_{T D H 3}-A D H 3-\mathrm{T}_{A D H 1}-\mathrm{P}_{H X T 7 m 6}-H P O-\mathrm{T}_{T P I 1}-\mathrm{P}_{H X T 7}-A t C P R-\mathrm{T}_{T P I I}$ & This study \\
\hline $\mathrm{p} 414-\mathrm{H}_{\mathrm{m} 6} \mathrm{~A}$ & $\mathrm{p} 414$ derived, containing $\mathrm{P}_{H X T 7 m 6}-H P O-\mathrm{T}_{T P I I}-\mathrm{P}_{H X T 7}-A t C P R-\mathrm{T}_{T P I 1}$ & This study \\
\hline p426 & $\mathrm{p} 426-\mathrm{P}_{S N R 52}-\mathrm{T}_{S U P 4}, U R A 3, A m p^{\mathrm{R}}$ & Addgene \\
\hline p426-SED1 & P426 derived, p426- $\mathrm{P}_{S N R 52-\mathrm{gRNA}}$ SED1-T $\mathrm{T}_{S U P 4}$ & This study \\
\hline p426-yp1062w & P426 derived, p426- $\mathrm{P}_{S N R 52-\mathrm{gRNA} . y \mathrm{p} 1062 \mathrm{w}-\mathrm{T}_{S U P 4}}$ & This study \\
\hline $\mathrm{p} 426-L E U 2$ & $\mathrm{P} 426$ derived, $\mathrm{p} 426-\mathrm{P}_{S N R 52-\mathrm{gRNA}}$ LEU2- $\mathrm{T}_{S U P 4}$ & This study \\
\hline
\end{tabular}


Table S3 Strains used in this study

\begin{tabular}{|c|c|c|c|}
\hline Strain & Parent strain & Plasmids/genotype & Source \\
\hline \multicolumn{4}{|l|}{ E. coli } \\
\hline DH5 $\alpha$ & & $\begin{array}{l}\mathrm{F}^{-} \varphi 80 \text { lac } \mathrm{Z} \Delta \mathrm{M} 15 \Delta(\text { lacZYA-argF) } \mathrm{U} 169 \text { recA1 end } \mathrm{A} 1 \text { hsd } \mathrm{R} 17 \\
\left(\mathrm{r}_{\mathrm{K}}^{-}, \mathrm{m}_{\mathrm{K}}^{+}\right) \text {phoA supE44 } \lambda^{-} \text {thi-1 gyrA96 relA1 }\end{array}$ & Invitrogen \\
\hline \multicolumn{4}{|l|}{ S. cerevisiae } \\
\hline CEN.PK2-1Ca & & MATa trp1-289 leu2-3 leu2-112 ura3-52 his3Al & ATCC $®$ MYA- $1108^{\mathrm{TM}}$ \\
\hline BY4741 & & MATa his $3 \Delta 1$ leu $2 \Delta 0$ met $15 \Delta 0$ ura $3 \Delta 0$ & Invitrogen \\
\hline BJ5464 & & 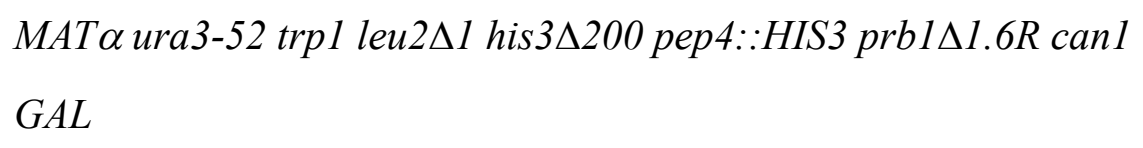 & ATCC® $208288^{\mathrm{TM}}$ \\
\hline PK2-H & CEN.PK2-1Ca & p352-H GGGSA & This study \\
\hline PK2-HGGGStA & CEN.PK2-1Ca & p352-H GGGStA & This study \\
\hline PK2-HEAAAKtA & CEN.PK2-1Ca & p352-HEAAAKtA & This study \\
\hline PK2-HA & CEN.PK2-1Ca & p352-HA & This study \\
\hline $\mathrm{PK} 2 \Delta s e d 1$ & CEN.PK2-1Ca & $\operatorname{sed} 1:: m u t$ & This study \\
\hline PK2-GUS & CEN.PK2-1Ca & $\mathrm{p} 352-G U S$ & This study \\
\hline $\mathrm{PK} 2 \Delta s e d 1-G U S$ & CEN.PK2-1Ca & sed1::mut, p352-GUS & This study \\
\hline
\end{tabular}




\begin{tabular}{|c|c|c|c|}
\hline PK2-C & CEN.PK2-1Ca & sed1::mut, p414-GUS & This study \\
\hline PK2-m1 & CEN.PK2-1Ca & sed1::mut, p414-GUS1 & This study \\
\hline PK2-m2 & CEN.PK2-1Ca & sed1::mut, p414-GUS2 & This study \\
\hline PK2-m3 & CEN.PK2-1Ca & sed1::mut, p414-GUS3 & This study \\
\hline PK2-m4 & CEN.PK2-1Ca & sed1::mut, p414-GUS4 & This study \\
\hline PK2-m5 & CEN.PK2-1Ca & sed1::mut, p414-GUS5 & This study \\
\hline PK2-m6 & CEN.PK2-1Ca & sed1::mut, p414-GUS6 & This study \\
\hline PK2-m7 & CEN.PK2-1Ca & sed1::mut, p414-GUS7 & This study \\
\hline PK2-m8 & CEN.PK2-1Ca & sed1::mut, p414-GUS8 & This study \\
\hline PK2-HA 1 & CEN.PK2-1Ca & $\mathrm{p} 352-\mathrm{HA}_{\mathrm{m} 1}$ & This study \\
\hline PK2-HA 2 & CEN.PK2-1Ca & $\mathrm{p} 352-\mathrm{HA}_{\mathrm{m} 2}$ & This study \\
\hline PK2-HA 3 & CEN.PK2-1Ca & $\mathrm{p} 352-\mathrm{HA}_{\mathrm{m} 3}$ & This study \\
\hline PK2-HA 4 & CEN.PK2-1Ca & $\mathrm{p} 352-\mathrm{HA}_{\mathrm{m} 4}$ & This study \\
\hline PK2-HA 5 & CEN.PK2-1Ca & $\mathrm{p} 352-\mathrm{HA}_{\mathrm{m} 5}$ & This study \\
\hline PK2-HA 6 & CEN.PK2-1Ca & $\mathrm{p} 352-\mathrm{HA} \mathrm{m} 6$ & This study \\
\hline PK2-HA 7 & CEN.PK2-1Ca & $\mathrm{p} 352-\mathrm{HA}_{\mathrm{m} 7}$ & This study \\
\hline PK2-HA & CEN.PK2-1Ca & $\mathrm{p} 352-\mathrm{HA}_{\mathrm{m} 8}$ & This study \\
\hline
\end{tabular}




\begin{tabular}{|c|c|c|c|}
\hline $\mathrm{PK} 2-\mathrm{H}_{\mathrm{m} 1} \mathrm{~A}$ & CEN.PK2-1Ca & $\mathrm{p} 352-\mathrm{H}_{\mathrm{m} 1} \mathrm{~A}$ & This study \\
\hline $\mathrm{PK} 2-\mathrm{H}_{\mathrm{m} 2} \mathrm{~A}$ & CEN.PK2-1Ca & $\mathrm{p} 352-\mathrm{H}_{\mathrm{m} 2} \mathrm{~A}$ & This study \\
\hline $\mathrm{PK} 2-\mathrm{H}_{\mathrm{m} 3} \mathrm{~A}$ & CEN.PK2-1Ca & $\mathrm{p} 352-\mathrm{H}_{\mathrm{m} 3} \mathrm{~A}$ & This study \\
\hline $\mathrm{PK} 2-\mathrm{H}_{\mathrm{m} 4} \mathrm{~A}$ & CEN.PK2-1Ca & $\mathrm{p} 352-\mathrm{H}_{\mathrm{m}} \mathrm{A}$ & This study \\
\hline $\mathrm{PK} 2-\mathrm{H}_{\mathrm{m} 5} \mathrm{~A}$ & CEN.PK2-1Ca & $\mathrm{p} 352-\mathrm{H}_{\mathrm{m}} \mathrm{A}$ & This study \\
\hline $\mathrm{PK} 2-\mathrm{H}_{\mathrm{m} 6} \mathrm{~A}$ & CEN.PK2-1Ca & $\mathrm{p} 352-\mathrm{H}_{\mathrm{m} 6} \mathrm{~A}$ & This study \\
\hline PK2-H $\mathrm{H}_{\mathrm{m} 7} \mathrm{~A}$ & CEN.PK2-1Ca & $\mathrm{p} 352-\mathrm{H}_{\mathrm{m} 7} \mathrm{~A}$ & This study \\
\hline $\mathrm{PK} 2-\mathrm{H}_{\mathrm{m} 8} \mathrm{~A}$ & CEN.PK2-1Ca & $\mathrm{p} 352-\mathrm{H}_{\mathrm{m} 8} \mathrm{~A}$ & This study \\
\hline PK2-tC & CEN.PK2-1Ca & $\operatorname{erg} 9:: \Delta-220--176$ rox $1:: m u t, \mathrm{p} 352-\mathrm{tC}$ & This study \\
\hline $\mathrm{PK} 2-\mathrm{tC} / \mathrm{AH}_{\mathrm{m} 6} \mathrm{~A}$ & CEN.PK2-1Ca & 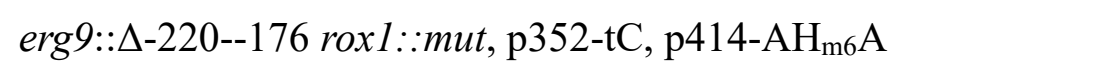 & This study \\
\hline $\mathrm{PK} 2-\mathrm{AtC} / \mathrm{H}_{\mathrm{m}} \mathrm{A}$ & CEN.PK2-1Ca & 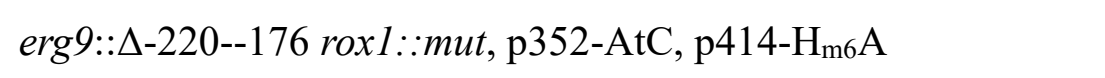 & This study \\
\hline $\mathrm{PK} 2-\mathrm{AtEC} / \mathrm{H}_{\mathrm{m} 6} \mathrm{~A}$ & CEN.PK2-1Ca & 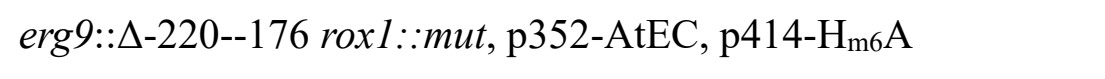 & This study \\
\hline PK2RI-AtC/H ${ }_{\mathrm{m} 6} \mathrm{~A}$ & & 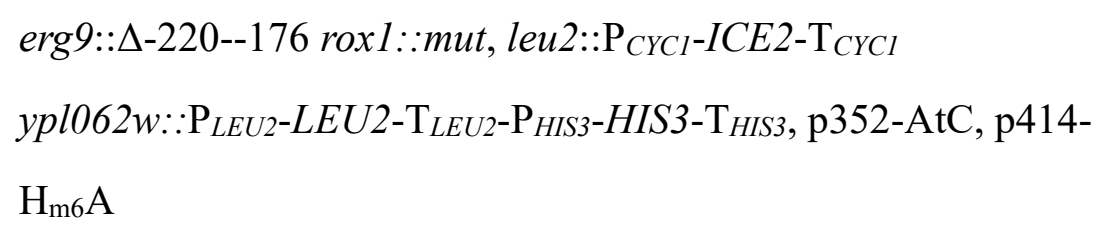 & This study \\
\hline $\mathrm{BY} \Delta s e d 1$ & BY4741 & sed1::mut & This study \\
\hline BY-GUS & BY4741 & p352-GUS & This study \\
\hline
\end{tabular}




\begin{tabular}{|c|c|c|c|}
\hline BY $\Delta s e d 1-G U S$ & BY4741 & sed1::mut, p352-GUS & This study \\
\hline $\mathrm{BJ} \Delta \operatorname{sed} 1$ & BJ5464 & sed1::mut & This study \\
\hline $\mathrm{BJ}-G U S$ & BJ5464 & $\mathrm{p} 352-G U S$ & This study \\
\hline $\mathrm{BJ} \Delta \operatorname{sed} 1-G U S$ & BJ5464 & sed1::mut, p352-GUS & This study \\
\hline
\end{tabular}

